\title{
LLNL-TR-609573
}

LAWRENCE LIVERMORE N A TIO NAL LABORATORY

Opening The NIF Archive To The Scientific User Community

A. Casey

January 4, 2013 
This document was prepared as an account of work sponsored by an agency of the United States government. Neither the United States government nor Lawrence Livermore National Security, LLC, nor any of their employees makes any warranty, expressed or implied, or assumes any legal liability or responsibility for the accuracy, completeness, or usefulness of any information, apparatus, product, or process disclosed, or represents that its use would not infringe privately owned rights. Reference herein to any specific commercial product, process, or service by trade name, trademark, manufacturer, or otherwise does not necessarily constitute or imply its endorsement, recommendation, or favoring by the United States government or Lawrence Livermore National Security, LLC. The views and opinions of authors expressed herein do not necessarily state or reflect those of the United States government or Lawrence Livermore National Security, LLC, and shall not be used for advertising or product endorsement purposes.

This work performed under the auspices of the U.S. Department of Energy by Lawrence Livermore National Laboratory under Contract DE-AC52-07NA27344. 


\section{X.XX Opening The NIF Archive To The Scientific User}

\section{Community}

\section{Executive Summary}

The National Ignition Facility (NIF) is the world's largest and most energetic laser, providing a research center to study inertial confinement fusion ignition and explore matter at extreme energy densities and pressures. With each experiment potentially generating hundreds of gigabytes of data, it is imperative that scientists be capable of interacting with the NIF Archive efficiently, visually and with tools that facilitate further experimental analysis. To do this, the Shot Data Systems team provides interactive, webbased dashboards, a virtual file system interface, a scripting language for programmatic data integration, and an experimental Wiki to support collaboration.

\section{Progress in 2012}

The NIF Archive is the permanent repository for experimental results produced by a NIF shot. The archive is an Oracle database with software interfaces written on top of a low-level Java content management API. Defining classes in the archive using the API ensures that the objects will always be created unambiguously, in the correct structures for the class of data and that they will contain enough meta-data to be able to describe the object through it attributes. This structure provides the basis on which the new features have been built.

Experiment dashboards are a custom assembly of interactive browser widgets, like portlets, that render a set of objects from the archive. A dashboard could display graphs of laser power, a camera image and tables of laser energy using the widgets that display graphs, images and tables. As each widget knows the type and attributes of the object it 
needs to display, no coding is required in order to display the data (Figure a). It is a matter of defining the layout of the widgets on the dashboard and then assigning the object to be displayed to the relevant widget. This approach to visualization has reduced the development time to visualize data in the archive from many weeks to a matter of hours.

Two new features have been added to the archive in order to support direct access to the data in the archive. Firstly, the archive has implemented a version of the Web Distributed Authoring and Versioning (WebDAV) protocol that allows users to browse the files in the archive as if they were navigating them in a directory structure either in a web browser or as a drive on the client computer. As well as browsing, the addition of a Groovy / Velocity based scripting language allows the users to programmatically package data from the archive for later download and analysis. The scripts can be run as needed or set up to execute following an event such as post shot analysis. The hierarchical nature of the archive enforced by the API facilitated these forms of access and both of these features have greatly simplified the task of extracting data from the database for the user.

Finally, as research is a collaborative effort, the archive is now integrated with the experimental Wiki. When a scientist sees something of note in the archive, they can post a comment directly to the Wiki that can then be read by the rest of the team in real time. This eliminates the need to have both Wiki and Archive sessions running concurrently. 


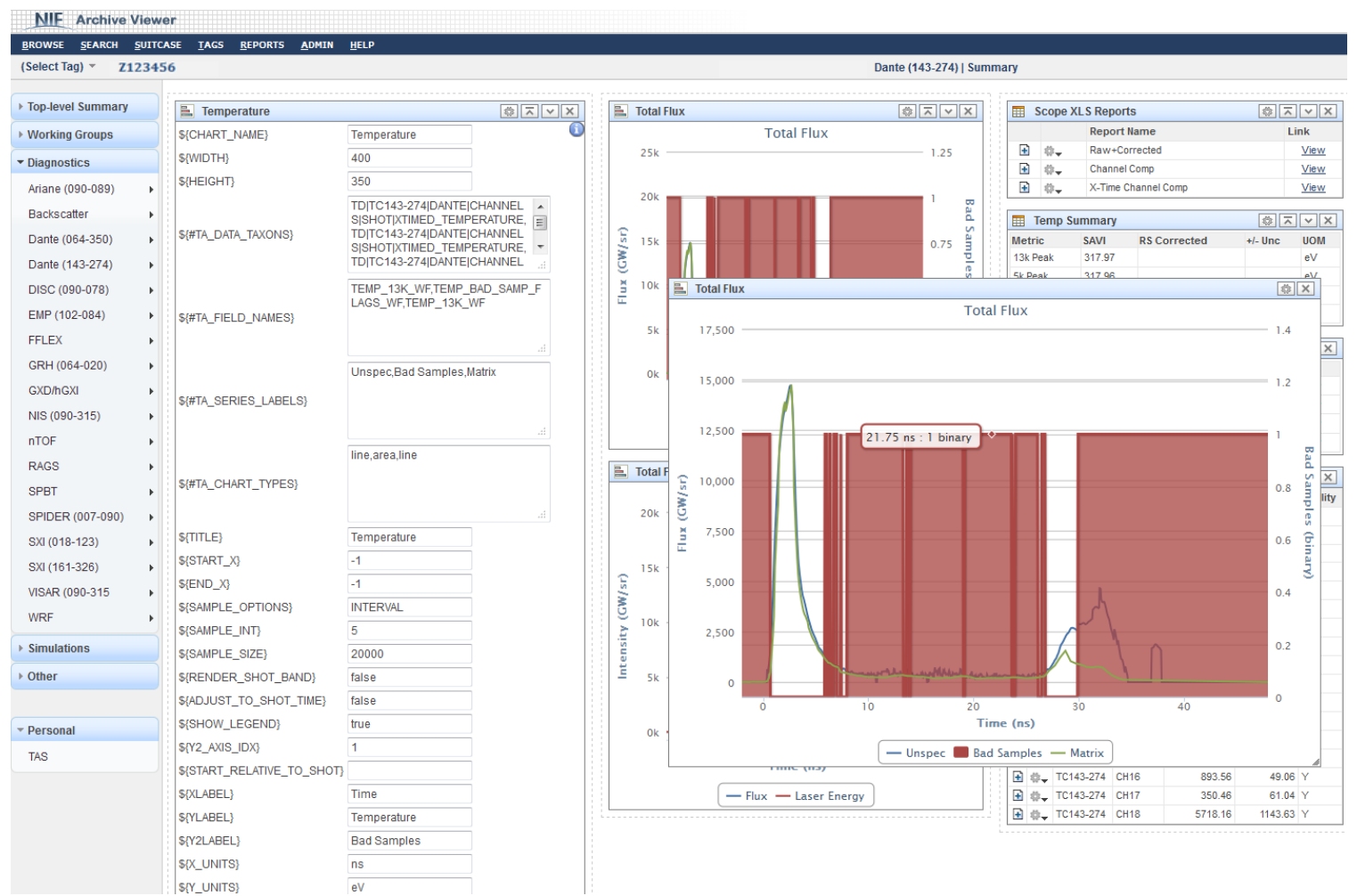

\section{Word-Count Reminder:}

1-page article: 1 figure $=550$ words

2-page article: 2 figures $=1000$ words; 1 figure $=$ (2-page article should have 2 figures)

The text below this box is not included in the word count.

\section{Review: DO NOT SUBMIT ARTICLE WITHOUT COMPLETING THIS SECTION}

Name of Derivative Classifier or DUSA:

Date Reviewed:

Topics/Guides:

\section{Contact information}

Name, casey20@1lnl.gov

External Web site (if applicable), website.llnl.gov

\section{Captions}

Figure a. Examples of Archive Viewer portlets showing their data driven configuration and interactivity 